\title{
Triple primary cancer of the head and neck, skin and prostate: A case report and literature review
}

\author{
NOBUYUKI MARUYAMA ${ }^{1,2}$, TOSHIYUKI NAKASONE ${ }^{2}$, OSAO ARAKAKI $^{3}$, HIROFUMI MATSUMOTO $^{4}$, \\ TESSHO MARUYAMA $^{1,2}$, AKIRA MATAYOSHI ${ }^{2}$, TAKAHIRO GOTO ${ }^{2}$, SEIICHI SAITO $^{5}$, \\ NAOKI YOSHIMI ${ }^{4,6}$, AKIRA ARASAKI ${ }^{1,2}$ and KAZUHIDE NISHIHARA ${ }^{1,2}$ \\ ${ }^{1}$ Department of Oral and Maxillofacial Functional Rehabilitation, Graduate School of Medicine, \\ University of The Ryukyus; Departments of ${ }^{2}$ Oral and Maxillofacial Surgery, ${ }^{3}$ Dermatology and \\ ${ }^{4}$ Pathology, University Hospital of The Ryukyus; Departments of ${ }^{5}$ Urology, and ${ }^{6}$ Pathology and Oncology, \\ Graduate School of Medicine, University of The Ryukyus, Okinawa 903-0215, Japan
}

Received January 20, 2018; Accepted June 13, 2018

DOI: $10.3892 / \mathrm{ol} .2018 .9294$

\begin{abstract}
Second primary cancer (SPC) is an important prognostic factor for patients with head and neck cancer (HNC); therefore, the association between the prognosis and development of SPC has been well-reported. The use of 2-[ $\left.{ }^{18} \mathrm{~F}\right]$-fluoro-2-deoxy-D-glucose-positron emission tomography (FDG-PET) is valuable to examine cancer stage, evaluate treatment responses and investigate suspected relapses or metastases. In the present study, the case of a male patient who was diagnosed with three primary cancer types, including well to moderately differentiated squamous cell carcinoma (SCC) of the mandible, axillary cutaneous poorly differentiated SCC and prostate adenocarcinoma, was described. Among these, mandible cancer was the first diagnosed when the patient was 70 years of age. Synchronous skin and prostate cancer (PRC) types then developed 3 years later. To the best of our knowledge, this is the first report of the aforementioned combination of cancer types. Postoperative FDG-PET was not performed as no lesions of recurrence or metastases of mandible cancer were found. Three years later,
\end{abstract}

Correspondence to: Dr Tessho Maruyama, Department of Oral and Maxillofacial Functional Rehabilitation, Graduate School of Medicine, University of The Ryukyus, 207 Uehara, Nishihara, Okinawa 903-0215, Japan

E-mail: h075324@eve.u-ryukyu.ac.jp

Abbreviations: SPC, second primary cancer; FDG-PET, 2-[18F]-fluoro-2-deoxy-D-glucose-positron emission tomography; SEER, Surveillance, Epidemiology and End Results; HNC, head and neck cancer; PC, primary cancer; SCC, squamous cell carcinoma; $\mathrm{AC}$, adenocarcinoma; CT, computed tomography; MRI, magnetic resonance imaging; SUVmax, maximum standardized uptake value; PSA, prostate-specific antigen; PRC, prostate cancer

Key words: head and neck cancer, second primary, skin cancer, prostate cancer, 2-[18F]-fluoro-2-deoxy-D-glucose-positron emission tomography, multiple primary neoplasms the PRC was asymptomatic and was incidentally detected by FDG-PET performed for a preoperative evaluation of skin cancer. It was indicated that FDG-PET could be utilized in patients with HNC due to there being no accurate FDG-PET protocol to detect SPC over a long-term follow-up.

\section{Introduction}

With progression in cancer treatment, the survival rate of patients has increased globally in $2012(1,2)$. In the USA, the number of people with a cancer diagnosis was $~ 14.5$ million in 2014 (3). Based on the Surveillance, Epidemiology and End Results (SEER) program, cancer survivors have a higher risk of second primary cancer (SPC) compared with the general population (4). Despite the medical progression achieved and the advances in locoregional control, continual SPC is one of the factors that impede the improvement of survival rate of patients with cancer (5); therefore, SPC is an important prognostic factor for patients with head and neck cancer (HNC) (6). Furthermore, SPC more frequently develops following $\mathrm{HNC}$, compared with the general population $(5,7)$. 2-[18F]-fluoro-2-deoxy-D-glucose-positron emission tomography (FDG-PET) is primarily used to examine lymph nodes, the initial stage of HNC, treatment responses, unknown primary cancer (PCs) types and relapses or metastases (8-11); however, there is no accurate FDG-PET protocol to detect SPC over a long-term follow-up period subsequent to HNC treatment. In the present study, a rare case of triple PC, including well to moderately differentiated squamous cell carcinoma (SCC) of the mandible, axillary cutaneous poorly differentiated SCC and adenocarcinoma (AC) of the prostate, was described. The prostate tumor was incidentally detected by FDG-PET. The aim of the present study was to discuss the necessity of FDG-PET as a protocol of postoperative follow-up.

\section{Case report}

Written informed consent was obtained from the patient for the publication of this case report and the accompanying images. The report was submitted for ethical review to the 
Ethics Committee of the University of the Ryukyus (Okinawa, Japan), which waived the requirement for review per institutional protocol due to the study not containing content that requires ethical approval. The Ethics Committee approved the submission and publication of the manuscript.

A 70-year-old man was evaluated and treated for cancer of the right mandible in September 2012 at the Department of Oral and Maxillofacial Surgery, University Hospital of the Ryukyus (Okinawa, Japan). The disease was initially considered to be benign or an inflammation in the radiolucent area around the wisdom teeth, and the patient underwent extraction of the right third molar and curettage (with histological test) at a different clinic (Nanbu Tokushukai Hospital, Okinawa, Japan); however, the histopathological examination at this clinic demonstrated well to moderately differentiated SCC, according to the World Health Organization Classification of Tumours (12) (Fig. 1). Histopathological examination was conducted with hematoxylin and eosin staining. In brief, resected tissues were fixed in $20 \%$ formalin for $\sim 24 \mathrm{~h}$ at room temperature. Subsequently, slides were washed with xylene for $9 \mathrm{~min}$, then dexylened with $100,95,80$ and $70 \%$ ethanol for 20,10, 10 and 10 times, respectively. Slides were then stained with hematoxylin for $10 \mathrm{~min}$, and washed with tap water for $3 \mathrm{~min}$. Subsequently, slides were washed with $0.5 \%$ hydrochloric acid alcohol once and washed with tap water for $5 \mathrm{~min}$. Following this, slides were stained with eosin for 6 min and slides were dehydrated with 95 and 100\% ethanol for 10 and 40 times, respectively. Additionally, slides were immersed in xylene 30 times and cover glass was placed on the slides. All of the methods were at room temperature, and then examined using a light microscope at x200 magnification. Subsequently, the patient was referred to the University Hospital of the Ryukyus. Physical examination indicated slight swelling of the gum around the extraction socket. No specific lesion was located in the mouth, and no palpable lymph node enlargement was evident in the head or neck region. The patient had no history of cancer and had never undergone radiation therapy or chemotherapy. Additionally, the patient had been a moderate smoker in his 20 s and was a moderate drinker. The family history included gastric cancer in two brothers. The patient also suffered from well-controlled hypertension, reflux esophagitis, hyperuricemia, sensorineural hearing loss, cerebral infarction and dyslipidemia.

Contrast-enhanced computed tomography (CT), contrast-enhanced magnetic resonance imaging (MRI) and FDG-PET were used to examine the mandible cancer stage. The CT results demonstrated bone resorption in the right mandible and tumor invasion was suspected (Fig. 2), whereas no lesions were observed in the cervical lymph nodes, lungs, bones or liver. The MRI results indicated high-signal intensity around the margins of the curettaged area; however, no other lesions were located in the head and neck region. FDG-PET was used to examine the stage of SCC and revealed increased FDG uptake [maximum standardized uptake value (SUVmax), 11.9] in the right mandible. Additionally, no other potential cancer areas were located in the rest of the body. An upper gastrointestinal endoscopy demonstrated no abnormal data. As a result, the patient was diagnosed with mandibular SCC (T4NOM0, stage IVa), according to the Union for International Cancer Control Tumor-Node-Metastasis classification, 7th edition (13).
The patient received 6 cycles of bleomycin chemotherapy preoperatively (total dose, $90 \mathrm{mg}$ ), as described previously $(14,15)$. In October 2012, the patient underwent segmental mandibulotomy and reconstructive grafting (non vascularized bone grafting), ipsilateral supraomohyoid neck dissection and tracheotomy. A pathological examination demonstrated SCC of the submaxilla, with venous invasion but no lymphatic invasion. Furthermore, bone invasion was present, but the surgical margins were negative. Cervical lymph nodes exhibited no evidence of cancer. Following surgery, a salivary fistula of the parotid region persisted. The patient then underwent postoperative radiation (total dose, $20 \mathrm{~Gy}$ in 10 fractions over $\sim 3$ weeks). Resolution of the salivary fistula using low-dose radiation therapy was attempted $(16,17)$, and notably, the fistula was successfully resolved. Although a number of studies have reported a lower success rate of radiotherapy following the reconstruction of a non-vascularized bone graft $(18,19)$, the radiotherapy for those patients is not contraindicated in the National Comprehensive Cancer Network guideline (20); therefore, a low-dose radiation was selected to resolve the salivary fistula of the parotid region (17). Surgical site infection did occur, and the grafted bone and titan plate were surgically removed 4 months after the implantation. Subsequently, no clinical or radiological signs of mandible cancer recurrence/metastasis or infection in the head and neck region or the lungs were observed.

The patient developed skin cancer (second type) and prostate cancer (PRC) (third type) 3 years after the mandible (first type) cancer treatment. A nodule on the left axillary cutaneous lesion was noted by the patient in July 2015. In April 2016, the nodule (18x10x7 mm in size) was resected at a different hospital (Chibana Clinic, Okinawa, Japan) and pathologically diagnosed as poorly differentiated SCC of the skin, according to the World Health Organization Classification of Tumours (21). A pathological examination demonstrated poorly differentiated SCC, with positive margins (data not shown). Pathological examination was conducted with hematoxylin and eosin staining. In brief, resected tissues were fixed in $10 \%$ formalin for $24 \mathrm{~h}$ at room temperature. Subsequently, slides were washed with xylene for $15 \mathrm{~min}$, then dexylened with 100, 80 and 50\% ethanol for 2, 1 and $1 \mathrm{~min}$, respectively. Subsequently, slides were washed with tap water for $2 \mathrm{~min}$. Following this, slides were washed with distilled water for $30 \mathrm{sec}$. Slides were then stained with hematoxylin for $4 \mathrm{~min}$ and washed with tap water for 6 min. Additionally, slides were washed with distilled water for $30 \mathrm{sec}$. Subsequently, slides were stained with eosin for $3 \mathrm{~min}$ and slides were dehydrated with 100\% ethanol for 4 min. Following this, slides were immersed in xylene for $8 \mathrm{~min}$ and cover glass was placed on the slides. All of the methods were at room temperature, and then examined using a light microscope at x200 magnification. Subsequently, the patient was referred to University Hospital of the Ryukyus for further examination and treatment. Contrast-enhanced CT and FDG-PET were performed to evaluate the skin cancer stage. There was no accumulation in the axillary skin, axillary lymph nodes, lungs, and head and neck region; however, FDG uptake was observed in the prostate (SUVmax, 4.61) (Fig. 3). The patient had a prostate-specific antigen (PSA) level of $19.87 \mathrm{ng} / \mathrm{ml}$ (normal range, $0-3.53 \mathrm{ng} / \mathrm{ml}$ ). Due to the absence of clinical symptoms 


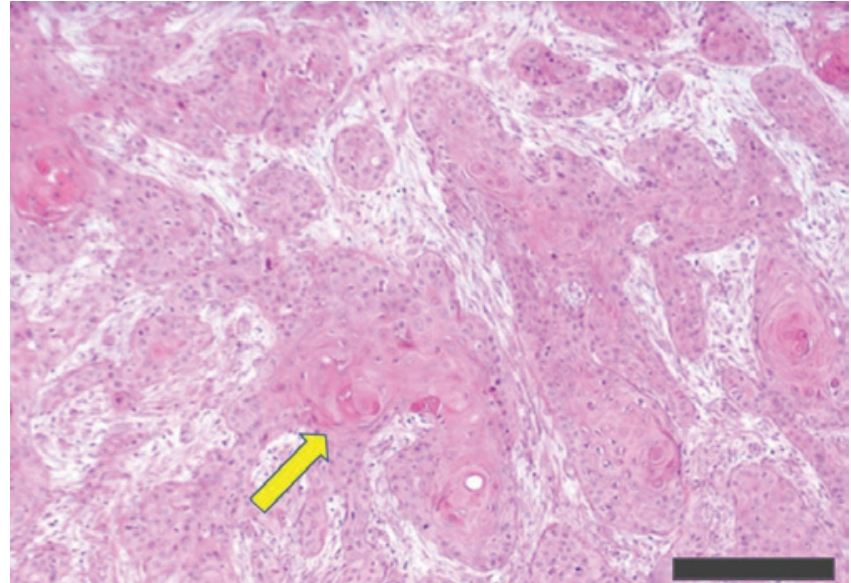

Figure 1. Histological examination of the mandible tissue by hematoxylin and eosin staining. Atypical squamous cells and cancer pearls are observed in the alveolar bone of the mandible, with intercellular bridges (arrow), which are characteristics of well-differentiated squamous cell carcinoma Magnification, $\mathrm{x} 200$; scale bar, $100 \mu \mathrm{m}$.

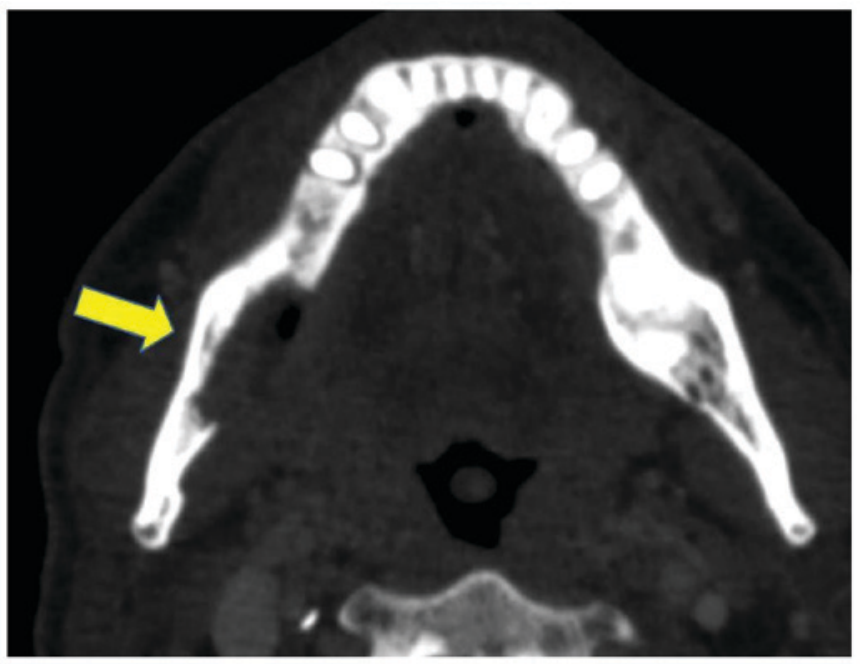

Figure 2. Contrast-enhanced computed tomography results indicating resorption in the right mandible and suspected tumor invasion (arrow) (axial section).

of 'non-head and neck, lung and esophagus cancer', the PSA or carcinoembryonic antigen test was not performed prior to the PET examination of the skin cancer. Notably, the SPC incidence of this cancer type (non-head and neck, lung and esophagus cancer) is low in patients with HNC (22). Additional resection of skin (second) cancer was first performed in June 2016 at the University Hospital of the Ryukyus and poorly differentiated SCC was resected. Pathological examination was conducted with hematoxylin and eosin staining. In brief, resected tissues were fixed in $10 \%$ formalin for $\sim 24 \mathrm{~h}$ at room temperature. Subsequently, slides were washed with xylene for $9 \mathrm{~min}$, then dexylened with 100,95 and $70 \%$ ethanol for 3, 1 and $1 \mathrm{~min}$, respectively. Subsequently, slides were washed with tap water for 3 min. Slides were then stained with hematoxylin for $4 \mathrm{~min}$, and washed with tap water for $3 \mathrm{~min}$. Following this, slides were dehydrated with $95 \%$ ethanol for $1 \mathrm{~min}$. Subsequently, slides were stained with eosin for $3 \mathrm{~min}$ and slides were washed with 95 and $100 \%$ ethanol for 2 and

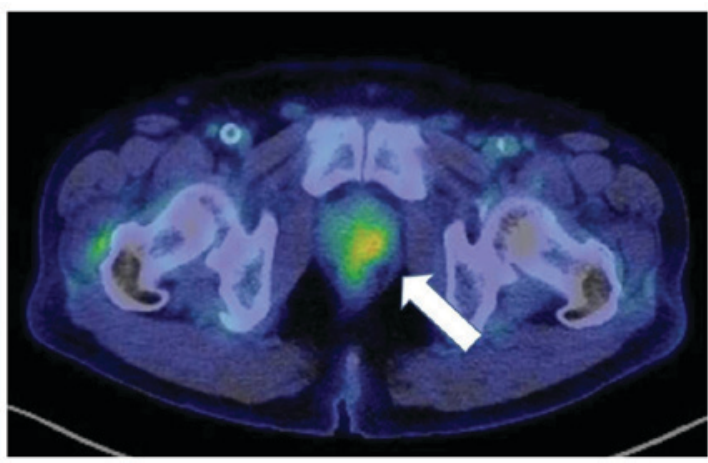

Figure 3. Preoperative use of 2-[18F]-fluoro-2-deoxy-D-glucose-positron emission tomography/computed tomography (axial section). Metabolic activity was detected in the prostate (maximum standardized uptake value, 4.61) (arrow).

8 min, respectively. Subsequently, slides were immersed in xylene for $12 \mathrm{~min}$ and cover glass was placed on the slides. All of the methods were at room temperature, and then examined using a light microscope at x100 magnification (Fig. 4). Following the resection of the second cancer, a guided percutaneous needle biopsy of the prostate lesion was performed in August 2016 at the University Hospital of the Ryukyus, which indicated a diagnosis of AC (Gleason score 4+5), according to Gleason and Mellinger (23). Laparoscopic prostatectomy and pelvic lymphadenectomy was then performed for the PRC (third type) in October 2016 at the University Hospital of the Ryukyus. Pathological data demonstrated positive pT4 (bladder) invasion (Fig. 5). Pathological examination was conducted with hematoxylin and eosin staining. In brief, resected tissues were fixed in $10 \%$ formalin for $\sim 24 \mathrm{~h}$ at room temperature. Subsequently, slides were washed with xylene for 9 min, then dexylened with 100,95 and $70 \%$ ethanol for 3,1 and $1 \mathrm{~min}$, respectively. Subsequently, slides were washed with tap water for $3 \mathrm{~min}$. Slides were then stained with hematoxylin for $4 \mathrm{~min}$ and washed with tap water for $3 \mathrm{~min}$. Following this, slides were dehydrated with $95 \%$ ethanol for $1 \mathrm{~min}$. Subsequently, slides were stained with eosin for $3 \mathrm{~min}$ and slides were washed with 95 and $100 \%$ ethanol for 2 and $8 \mathrm{~min}$, respectively. Following this, slides were immersed in xylene for $12 \mathrm{~min}$ and cover glass was placed on the slides. All of the methods were at room temperature, and then examined using a light microscope at x200 magnification. Negative lymphatic (D2-40) and vascular (by Victoria blue-hematoxylin and eosin staining) invasion, positive perineural (S-100) invasion and positive margins, but no lymph node metastasis was found. Victoria blue-hematoxylin and eosin staining was conducted. In brief, resected tissues were fixed in $10 \%$ formalin for $\sim 24 \mathrm{~h}$ at room temperature. Subsequently, slides were washed with xylene for $9 \mathrm{~min}$, and then dexylened with 100, 95 and $70 \%$ ethanol for 6, 3 and $3 \mathrm{~min}$, respectively. Slides were then stained with Victoria blue overnight at room temperature, the remainder of Victoria blue was removed with $70 \%$ ethanol and then washed with tap water. Subsequently, slides were washed with xylene for $3 \mathrm{~min}$, and then washed with tap water for 3 min. Following this, slides were stained with hematoxylin for $3 \mathrm{~min}$. Subsequently, washed with tap water for $3 \mathrm{~min}$ and dehydrated with $95 \%$ ethanol 20 times. Following this, slides were stained with eosin for $3 \mathrm{~min}$, and then dehydrated with 


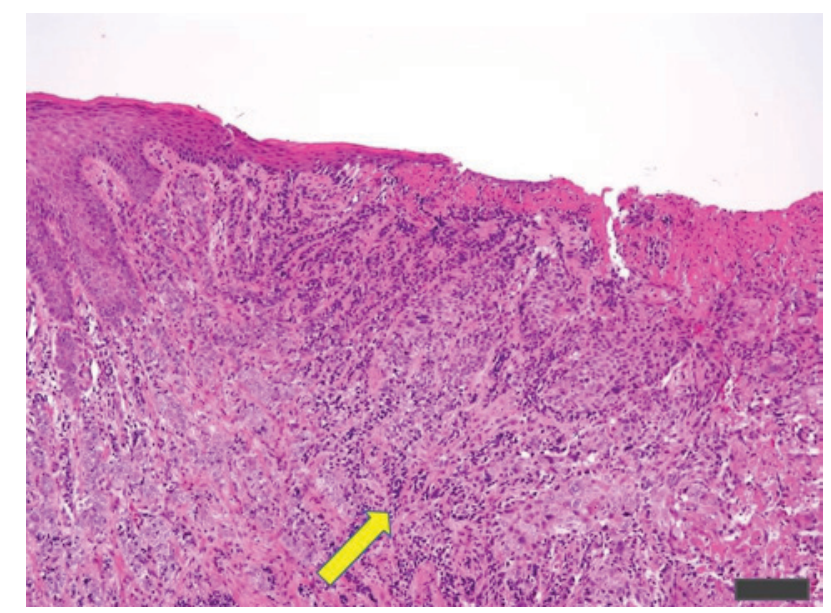

Figure 4. Histological examination of the skin tissue by hematoxylin and eosin staining. Irregular nests and cords composed of atypical squamous cell without keratinization infiltrate the dermis and subcutaneous tissue, and spindle-shaped cells are observed (arrow), which are characteristics of poorly differentiated squamous cell carcinoma. Magnification, x100; scale bar, $100 \mu \mathrm{m}$.

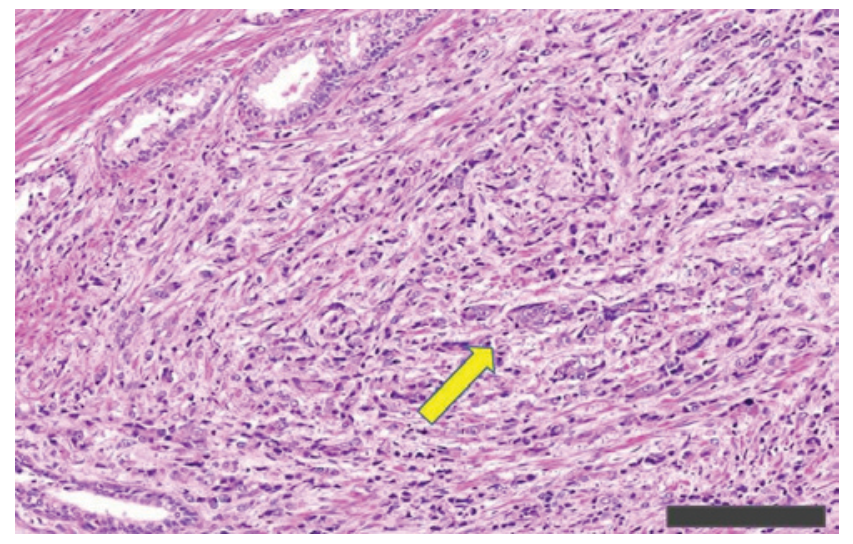

Figure 5. Histological examination of the prostate tissue by hematoxylin and eosin staining. Solid strands without glandular lumina invade the stroma (arrow). Magnification, x200; scale bar, $100 \mu \mathrm{m}$.

$100 \%$ ethanol for $4 \mathrm{~min}$. Slides were then immersed in xylene for $12 \mathrm{~min}$, and cover glass was placed on the slides. All of the methods were at room temperature, and then examined using a light microscope at x200 magnification. However, in February 2017, follow-up PSA indicated the high PSA level of $35.67 \mathrm{ng} / \mathrm{ml}$ (normal range, 0-3.53 ng/ml) was elevated, and subsequent CT and FDG-PET demonstrated recurrence of the PRC, accompanied with lymph node metastasis of the left axillary and pelvic regions, and suspected multiple liver metastases (the liver lesion was then examined by fine-needle biopsy in April 2017 and no cancerous lesions were histologically located). Axillary lymph node dissection was performed in July 2017, and the lymph nodes were histologically diagnosed as SCC metastases. The patient was treated with chemotherapy [from March 2017, docetaxel $\left(60 \mathrm{mg} / \mathrm{m}^{2}\right)$ was intravenously administered 10 times, and degarelix were administered subcutaneous injections into the abdominal region 14 times (240 mg once in March 2017 and then $80 \mathrm{mg} 13$ times)] for the liver, pelvic and prostate regions. The time between administration of the two drugs was 4 or 5 weeks. The lesions gradually diminished at the time of writing, therefore his prognosis cannot be stated, and further treatment of the chemotherapy (identical dose of docetaxel and degarelix) are planned. Based on the aforementioned lesions, the patient was diagnosed with 'triple PCs', according to the criteria by Warren and Gates (24), and the skin and PRCs were synchronous, according to the criteria by Moertel et al (25). The skin cancer (poorly differentiated SCC) was not considered the metastasis of the mandible cancer (well to moderately differentiated SCC), according to the criteria by Warren and Gates (24).

\section{Discussion}

There are two important points in the present report: Firstly, to the best of our knowledge, the combination of triple PCs (well-differentiated SCC of the mandible, axillary cutaneous poorly differentiated SCC and prostate AC) has not been previously reported; secondly, to detect SPC, we suggest that FDG-PET should be used for the long-term follow-up of patients with HNC.

Based on the aforementioned lesions, the patient in the present case was diagnosed with triple PCs according to the criteria suggested by Warren and Gates in 1932 (24), and the skin and PRCs were synchronous according to the criteria defined by Moertel et al in 1961 (25). A systematic literature search of PubMed (https://www.ncbi.nlm.nih.gov/pubmed/) and Google Scholar (https://scholar.google.co.jp/) articles published between 1932, when the SPC criteria was firstly defined (24), and 2017 was performed. The databases were searched using the following terminological combinations (one term used from each category): i) Any region of the head and neck, including oral cavity, oral, mouth, oral floor, tongue, lip, soft plate, gingiva, buccal, maxillary, mandibular, tonsil, neck, face, cheek, salivary grand, parotid gland, sublingual gland, submandibular gland, nose, nasal cavity, paranasal sinus, nasopharynx, larynx, oropharynx, mesopharynx, hypopharynx, glottis, thyroid, ear; ii) prostate; and iii) SPC terms, including second primary cancer, second primary malignancy, second primary tumor, multiple primary cancer, multiple primary neoplasm, multiple primary malignancies, multiple primary malignant, triple primary, triple cancer, quadruple primary, quadruple cancer, quintuple primary, quintuple cancer, sextuple primary, sextuple cancer, septuple primary, septuple cancer, octuple primary, octuple cancer, nonuple primary, or nonuple cancer. A report containing more than decuple primary cancer types, including PRC, could not be located. English articles were then searched using the aforementioned terms. This resulted in the identification of 23 cases of patients with multiple (triple or more) cancer types, including HNC and PRC (25-39) (Table I). The study by Gordon (26) was located by non-systematic literature research, using Google Scholar and the term 'synchronous primary carcinoma.' However, the current combination was not found. The most frequent sites of incident of SPC following HNC are the head and neck again, the lungs and the esophagus $(7,22)$; while SPC of the skin or prostate is uncommon. Although a number of risks of SPC have been suggested to date, the risk of SPC is unclear (40). As the combination of triple PCs in the present study was unique, the risk factors may have included the administration of preoperative chemotherapy (41) and radiation postoperatively for index mandible cancer $(41,42)$, the fact that 
Table I. Cases exhibiting triple or greater primary cancer of multiple types, including head and neck, and prostate cancer.

\begin{tabular}{|c|c|c|c|c|c|c|c|}
\hline \multirow[b]{2}{*}{ Author (year) } & \multicolumn{7}{|c|}{ Order of sites exhibiting the occurrence of multiple primary cancer } \\
\hline & First & Second & Third & Fourth & Fifth & Sixth & (Refs.) \\
\hline Gordon (1948) & PRC & Lung & Thyroid & & & & (26) \\
\hline Goodner and Watson (1956) & Soft plate & Esophagus & PRC & & & & $(27)$ \\
\hline \multirow[t]{7}{*}{ Moertel et $a l^{\mathrm{a}}$ (1961) } & Colon & Lip & PRC & & & & $(25)$ \\
\hline & Kidney & Mouth & PRC & & & & (25) \\
\hline & Lip & Lung & $\mathrm{PRC}$ & & & & (25) \\
\hline & Lung & PRC & Thyroid & & & & (25) \\
\hline & Lip & Skin & PRC & & & & (25) \\
\hline & Mouth & Mouth & PRC & & & & (25) \\
\hline & Lip & Mouth & PRC & & & & (25) \\
\hline \multirow[t]{2}{*}{ Bittorf et al (2001) } & PRC & Colorectum & Oral cavity & & & & $(28)$ \\
\hline & Thyroid & UB & PRC & & & & (28) \\
\hline Rho et al (2002) & Vocal cord & Bowen's disease & $\mathrm{PRC}$ & Laryngeal & & & $(29)$ \\
\hline Rai et al (2007) & PRC & Kidney & Thyroid & & & & $(30)$ \\
\hline Jaudah et al (2008) & Thyroid & PRC & Renal & & & & $(31)$ \\
\hline Yamashita et al (2010) & $\mathrm{PRC}$ & Gastric & Laryngeal & & & & $(32)$ \\
\hline \multirow[t]{2}{*}{ Salem et al (2012) } & PRC & Nasopharyngeal & Lung & & & & (33) \\
\hline & Renal & Nose & Auricle & PRC & Colon & & (33) \\
\hline Guven et al (2014) & Bladder & $\mathrm{PRC}$ & Thyroid & & & & (34) \\
\hline Mukaiyama et al (2014) & Glottis & Renal pelvis & UB & Oral floor & PRC & Esophagus & (35) \\
\hline Mohammed et al (2015) & PRC & UB & Thyroid & & & & $(36)$ \\
\hline Testori et al (2015) & Lung & Oropharynx & Large bowel & PRC & & & (37) \\
\hline Pastore et al (2015) & Kidney & Oropharynx & PRC & & & & $(38)$ \\
\hline Adel et al (2016) & Buccal & Lip & Gum & PRC & & & (39) \\
\hline Present case & Mandible & Axillary skin & $\mathrm{PRC}$ & & & & - \\
\hline
\end{tabular}

${ }^{\mathrm{a}}$ Order of incidence was unknown. PRC, prostate cancer; UB, urinary bladder.

the patient was a current drinker $(41,43)$, a family history of gastric cancer in two brothers (44) and finally, the patient being a former smoker $(43,45)$. The association between being a former smoker and secondary cancer risk in cancer survivors is unclear in the present case due to the cessation of smoking more than 40 years previously. Shiels et al (45) demonstrated that being a former smoker resulted in a higher SPC risk compared with the risk for those who had never smoked. Based on the aforementioned data, all factors for SPC risk in the current case should be carefully considered. Rose et al (6) analyzed 34,568 patients with non-metastatic SCC of HNC, based on SEER data, and reported that patients with $\mathrm{HNC}$ are at a high risk of SPC. The 5-year cumulative all-cause fatality rate, HNC-specific fatality rate, SPC fatality rate and non-cancer fatality rate were 51.3, 23.8, 14.6 and $13.0 \%$, respectively. Additionally, the 10-year cumulative rate of SPC mortality reached $20 \%$ (6). As a result, mortality due to SPC is high and persistent over a long term, and contributes to the poor prognosis of SCC in patients with HNC (6). Other previous studies have also indicated that even in long-term cancer survivors, SPC following HNC poses a high risk $(39,42,46)$. Furthermore, SPC more frequently develops in various sites in patients with HNC, compared with the SPC occurrence of the general population $(5,7)$; therefore, clinicians should give more focus to SPC following HNC treatment, and a more accurate protocol should be established.

We suggest that FDG-PET could be used for detecting SPCs in the long-term follow-up of patients with HNC. To date, there is no accurate protocol of FDG-PET for the follow-up of patients with HNC. In the present case, PRC (third type) was determined during the preoperative FDG-PET for skin (second type) cancer. The PRC (third type) was not determined during the first follow-up period of 3 years, between the mandible (first type) cancer treatment and the skin (second type) cancer occurrence. Contrast-enhanced CT was performed from the head to the lungs routinely during the follow-up subsequent to the HNC treatment; if FDG-PET had been performed as a routine follow-up tool of mandible cancer, the PRC may have been detected earlier. Cancer of the oral cavity and skin are relatively simple to detect due to the lesions being observed directly. Conversely, as PRC is asymptomatic in the early stages (47) and is an internal disease, it is frequently incidentally detected. In order to confirm the diagnosis method of PRC of those patients, the cases in Table I were further reviewed and the manner in which the subsequent PRC was detected is indicated $(28,29,31,33-35,37,38)$ (Table II). Of the 
Table II. Method of detecting subsequent PRC from cases of Table I.

\begin{tabular}{|c|c|c|c|c|}
\hline Author (year) & $\begin{array}{l}\text { Interval between } \\
\text { first cancer and } \\
\text { subsequent PRC }\end{array}$ & Method of detecting PRC & Subjective symptoms of PRC & (Refs.) \\
\hline Bittorf et al (2001) & 11 years & NA & NA & $(28)$ \\
\hline Rho et al (2002) & 3 years & $\begin{array}{l}\text { Serum tumor marker test } \\
\text { to rule out hidden cancer } \\
\text { following the diagnosis of } \\
\text { Bowen's disease }\end{array}$ & None & $(29)$ \\
\hline Jaudah et al (2008) & 10 years & Urinary symptom & Urinary symptom & $(31)$ \\
\hline Salem et al (2012) & 5 years & NA & NA & (33) \\
\hline Guven et al (2014) & Simultaneous & $\begin{array}{l}\text { Incidentally determined } \\
\text { in BC surgery }\end{array}$ & $\begin{array}{l}\text { Hematuria and frequent } \\
\text { urination, which were } \\
\text { considered to be a result } \\
\text { of } \mathrm{BC}\end{array}$ & (34) \\
\hline Mukaiyama et al (2014) & 3 years 9 months & $\begin{array}{l}\text { Incidentally determined in } \\
\text { recurrent BC surgery }\end{array}$ & None & $(35)$ \\
\hline Testori et al (2015) & Simultaneous & $\begin{array}{l}\text { Incidentally detected by } \\
\text { FDG-PET for suspected } \\
\text { lung cancer }\end{array}$ & None & $(37)$ \\
\hline Pastore et al (2015) & 7 months & $\begin{array}{l}\text { Serum tumor marker test } \\
\text { for follow-up after kidney } \\
\text { cancer treatment }\end{array}$ & None & $(38)$ \\
\hline Present case & 3 years 8 months & $\begin{array}{l}\text { Incidentally detected by } \\
\text { FDG-PET to determine the } \\
\text { preoperative axillary } \\
\text { cancer staging }\end{array}$ & None & - \\
\hline
\end{tabular}

PRC, prostate cancer; NA, not applicable; BC, bladder cancer; FDG-PET, 2-[18F]-fluoro-2-deoxy-D-glucose-positron emission tomography.

9 patients in which PRC was diagnosed, including the present case, PRC was detected by a serum test in 2 , incidentally by surgery of the other tumor in 2, by FDG-PET in 2 and by clinical symptoms in 1 (PRC detection in 2 patients was not described). Similar to the present case, Testori et al (37) incidentally detected lung cancer using FDG-PET (37). Notably, among the cases in Table II, $5 / 7$ patients presented with no subjective symptoms of PRC and were incidentally diagnosed with cancer. Additionally, in the SEER study, the incidence of secondary PRC within 1 year of HNC was only $0.3 \%$ among 26,258 male patients with oral and pharyngeal (tongue, mouth, tonsil, oropharyngeal and hypopharyngeal) cancer (4).

Patients with cancer should be carefully followed up in order to detect SPC for the following reasons: i) patients with cancer have a higher risk for SPC a long time period after PC compared with the general population (42); ii) as aforementioned, SPC more frequently develops in various sites in the body following HNC (5,7); iii) PRC should be detected at an early stage when it is asymptomatic, due to this condition demonstrating a poor prognosis at advanced stages (48) [for patients with localized stage PRC, the 5-year relative survival rate is $\sim 100 \%$; by contrast, for patients with advanced (distant) stage PRC, the rate declines to 28\% (49)]; and iv) PRC accounted for $20 \%$ of new cancer cases in males in the USA in 2016 (50). In the USA, among males, the most prevalent cancer in 2016 was PRC, which was recorded in 3.3 million cases (49); therefore, long-term follow-up to detect PRC following $\mathrm{HNC}$ treatment is required. Yamashita et al (43) routinely performed FDG-PET/CT scans once every 6-12 months for 5 years in the follow-up period following the initial cancer treatment of 434 patients with newly diagnosed $\mathrm{HNC}$, and determined that $12 \%$ of patients had synchronous SPC and $24 \%$ of patients had metachronous SPC. It is important to utilize FDG-PET for screening SPC during follow-up of HNC, as well as during preoperative cancer staging $(43,51)$. Compared with CT or MRI, PET/CT has the advantage of evaluating SPC not only at initial staging of first HNC (51), but also postoperative follow-up, similar to the present case, due to $\mathrm{PET} / \mathrm{CT}$ can evaluate the entire body range; however, there is no accurate protocol for detecting SPC for the long-term follow-up of HNC to date. FDG-PET/CT has an important role in the management of patients with HNC, in order to diagnose long-term surveillance of recurrence or metastasis (11). For patients with HNC, FDG-PET/CT is generally performed at $\geq 6$ months after the initial therapy (11); however, there are numerous studies regarding PET/CT that have reported a range of follow-up periods $(9,36,43,52)$, indicating that the 
optimal follow-up period has yet to be defined. In the present case, second primary PRC was detected 3 years after the initial HNC. Although PET/CT can be performed for extended follow-ups, it has certain disadvantages and risks, including the following: The scan may provide false-positive results (9); patients with diabetes mellitus cannot undergo PET/CT (53); PET/CT sensitivities depend on the body site (54); and finally, inflammatory lesions, metal artifacts or benign lesions can cause difficulties in performing PET/CT (55). Furthermore, the complications caused by the exposure to X-rays whilst performing PET/CT should be considered (53). Additionally, the decision to perform the examination differs among nations, indicating that clinicians should consider the characteristics of the health care system of each country.

There are several limitations in the present study, including the fact that the conclusions are based on a single case report, which limits the generalizability, and that the present combination of triple cancer was researched using PubMed and Google scholar, which are major search services, but other search engines were not used. For the present case, further studies may provide beneficial information for detecting SPCs, including PRC, following the treatment of HNC.

In conclusion, a rare case of triple PCs was described in the present study. We suggest that FDG-PET should be performed to detect hidden SPC, such as PRC, for the long-term follow-up of patients with HNC, particularly in cases where risk factors are present. SPC should be detected early in order to maximize positive patient outcomes.

\section{Acknowledgements}

The authors would like to thank Professor Kenzo Takahashi from the Department of Dermatology, Graduate School of Medicine, University of the Ryukyus (Okinawa, Japan) for his advice.

\section{Funding}

No funding was received.

\section{Availability of data and materials}

All data generated or analyzed during this study are included in this published article.

\section{Authors' contributions}

NM and TM acquired the data, performed the literature review and edited the manuscript. AA substantially contributed to the concept and design of the study. TN, OA, AM, TG, SS and KN acquired the data and contributed clinical advice. HM and AA revised the manuscript. $\mathrm{HM}$ and $\mathrm{NY}$ evaluated the specimens and gave histopathological advice. TM had a major role in writing the manuscript.

\section{Ethics approval and consent to participate}

The report was submitted for ethical review to the Ethics Committee of the University of the Ryukyus (Okinawa, Japan), which waived the requirement for review per institutional protocol due to the study not containing content that requires ethical approval. The Ethics Committee approved the submission and publication of the manuscript. Written informed consent was obtained from the patient for the publication of this case report and the accompanying images.

\section{Patient consent for publication}

Written informed consent was obtained from the patient for the publication of this case report and the accompanying images.

\section{Competing interests}

The authors declare that they have no competing interests.

\section{References}

1. Cakir A, Akgun Z, Fayda M and Agaoglu F: Comparison of three dimensional conformal radiation therapy, intensity modulated radiation therapy and volumetric modulated arc therapy for low radiation exposure of normal tissue in patients with prostate cancer. Asian Pac J Cancer Prev 16: 3365-3370, 2015.

2. IARC: GLOBOCAN 2012: Estimated Cancer Incidence, Mortality and Prevalence Worldwide in 2012. http://globocan. iarc.fr/Pages/fact sheets cancer.aspx. Accessed June 16, 2018.

3. Choi Y, Kim SY, Kim SH, Yang J, Park K and Byun Y: Inhibition of tumor growth by biodegradable microspheres containing all-trans-retinoic acid in a human head-and-neck cancer xenograft. Int J Cancer 107: 145-148, 2003.

4. Curtis RE, Freedman DM, Ron E, Ries LAG, Hacker DG, Edwards BK, Tucker MA and Fraumeni JF Jr: New malignancies among cancer survivors: SEER Cancer Registries, 1973-2000. National Cancer Institute. https://seer.cancer. gov/archive/publications/mpmono/MPMonograph_complete.pdf. Accessed September 18, 2017.

5. Morris LG, Sikora AG, Hayes RB, Patel SG and Ganly I: Anatomic sites at elevated risk of second primary cancer after an index head and neck cancer. Cancer Causes Control 22: 671-679, 2011.

6. Rose BS, Jeong JH, Nath SK, Lu SM and Mell LK: Population-based study of competing mortality in head and neck cancer. J Clin Oncol 29: 3503-3509, 2011.

7. Chuang SC, Scelo G, Tonita JM, Tamaro S, Jonasson JG, Kliewer EV, Hemminki K, Weiderpass E, Pukkala E, Tracey E, et al: Risk of second primary cancer among patients with head and neck cancers: A pooled analysis of 13 cancer registries. Int J Cancer 123: 2390-2396, 2008.

8. Plaxton NA, Brandon DC, Corey AS, Harrison CE, Karagulle Kendi AT, Halkar RK and Barron BJ: Characteristics and limitations of FDG PET/CT for imaging of squamous cell carcinoma of the head and neck: A comprehensive review of anatomy, metastatic pathways, and image findings. AJR Am J Roentgenol 205: W519-W531, 2015.

9. Al-Ibraheem A, Buck A, Krause BJ, Scheidhauer K and Schwaiger M: Clinical applications of FDG PET and PET/CT in head and neck cancer. J Oncol 2009: 208725, 2009.

10. Lonneux M, Hamoir M, Reychler H, Maingon P, Duvillard C, Calais G, Bridji B, Digue L, Toubeau M and Grégoire V: Positron emission tomography with $[18 \mathrm{~F}]$ fluorodeoxyglucose improves staging and patient management in patients with head and neck squamous cell carcinoma: A multicenter prospective study. J Clin Oncol 28: 1190-1195, 2010.

11. Manca G, Vanzi E, Rubello D, Giammarile F, Grassetto G, Wong KK, Perkins AC, Colletti PM and Volterrani D: (18) F-FDG PET/CT quantification in head and neck squamous cell cancer: Principles, technical issues and clinical applications. Eur J Nucl Med Mol Imaging 43: 1360-1375, 2016.

12. Takata $\mathrm{T}$ and Slootweg PJ: Tumours of the oral cavity and mobile tongue. In: World Health Organization (WHO) classification of head and neck tumours. El-Naggar AK, Chan JKC, Grandis JR, Takata T and Slootweg PJ (eds). 4th edition. IARC Press, Lyon, pp108-111, 2017.

13. Sobin LH, Gospodarowicz MK and Wittekind C: International Union Against Cancer. TNM classification of malignant tumors. 7th edition. Wiley-Blackwell, New York, NY, 2009. 
14. Licitra L, Grandi C, Guzzo M, Mariani L, Lo Vullo S, Valvo F, Quattrone $\mathrm{P}$, Valagussa $\mathrm{P}$, Bonadonna G, Molinari R and Cantù G: Primary chemotherapy in resectable oral cavity squamous cell cancer: A randomized controlled trial. J Clin Oncol 21: 327-333, 2003.

15. Yamamoto E, Kohama G, Sunakawa H, Iwai M and Hiratsuka H: Mode of invasion, bleomycin sensitivity, and clinical course in squamous cell carcinoma of the oral cavity. Cancer 51: 2175-2180, 1983

16. Mantsopoulos K, Goncalves M and Iro H: Transdermal scopolamine for the prevention of a salivary fistula after parotidectomy. Br J Oral Maxillofac Surg 56: 212-215, 2018

17. Shimm DS, Berk FK, Tilsner TJ and Coulthard SW: Low-dose radiation therapy for benign salivary disorders. Am J Clin Oncol 15: 76-78, 1992.

18. Maurer P, Eckert AW, Kriwalsky MS and Schubert J: Scope and limitations of methods of mandibular reconstruction: A long-term follow-up. Br J Oral Maxillofac Surg 48: 100-104, 2010.

19. Moura LB, Carvalho PH, Xavier CB, Post LK, Torriani MA, Santagata M and Chagas Júnior OL: Autogenous non-vascularized bone graft in segmental mandibular reconstruction: A systematic review. Int J Oral Maxillofac Surg 45: 1388-1394, 2016.

20. National Comprehensive Cancer Network: Head and Neck Cancers Version 1, 2018. https://www.nccn.org/professionals/physician_gls/pdf/head-and-neck.pdf. Accessed April 18, 2018.

21. Weedon D, Morgan MB, Gross C, Nagore E and Yu LL: Squamous cell carcinoma. In: Pathology and Genetics of Skin Tumours. LeBoit PE, Burg G and Weedon DAS (eds). IARC Press, Lyon, pp20-25, 2006.

22. Jain KS, Sikora AG, Baxi SS and Morris LG: Synchronous cancers in patients with head and neck cancer: Risks in the era of human papillomavirus-associated oropharyngeal cancer. Cancer 119: 1832-1837, 2013.

23. Gleason DF and Mellinger GT: Prediction of prognosis for prostatic adenocarcinoma by combined histological grading and clinical staging. J Urol 111: 58-64, 1974.

24. Warren S and Gates O: Multiple primary malignant tumors: A survey of the literature and a statistical study. Am J Cancer 16 $1358-1414,1932$

25. Moertel CG, Dockerty MB and Baggenstoss AH: Multiple primary malignant neoplasms. I. Introduction and presentation of data. Cancer 14: 221-230, 1961.

26. Gordon BS: Triple synchronous primary carcinoma. Arch Pathol (Chic) 45: 56-64, 1948.

27. Goodner JT and Watson WL: Cancer of the esophagus; its association with other primary cancers. Cancer 9: 1248-1252, 1956.

28. Bittorf B, Kessler H, Merkel S, Brückl W, Wein A, Ballhausen WG, Hohenberger W and Günther K: Multiple primary malignancies: An epidemiological and pedigree analysis of 57 patients with at least three tumours. Eur J Surg Oncol 27: 302-313, 2001

29. Rho NK, Choi SJ and Lee ES: A case of multiple Bowen's disease with squamous cell carcinoma of the larynx and adenocarcionoma of the prostate. J Dermatol 29: 516-521, 2002.

30. Rai RS, Deb P, Rai R, Gupta E and Panayach JS: Synchronous primary triple neoplasia (renal cell carcinoma and prostate cancer in combination with thyroid neoplasm). Report of an unusual case. Minerva Urol Nefrol 59: 451-454, 2007.

31. Jaudah AM, Kanaan HD and Hasan JF: Multiple primary malignancies of thyroid, kidneys and prostate: Synchronous and metachronous presentation in one patient. New Egypt J Med 39: 33-36, 2008.

32. Yamashita M, Jinbu Y, Hiratsuka M, Shinozaki Y, Itoh H and Kusama M: A case of quadruple primary cancer including lower lip cancer. Asian J Oral Maxillofac Surg 22: 172-174, 2010.

33. Salem A, Abu-Hijlih R, Abdelrahman F, Turfa R, Amarin R, Farah N, Sughayer M, Almousa A and Khader J: Multiple primary malignancies: Analysis of 23 patients with at least three tumors. J Gastrointest Cancer 43: 437-443, 2012.

34. Guven BC, Guzide Ayse GO and Ramazan S: Triple synchronous primary cancers of thyroid, bladder and prostate: A case report. Kuwait Med J 46: 62-64, 2014.

35. Mukaiyama Y, Suzuki M, Morikawa T, Mori Y, Takeshima Y, Fujimura T, Fukuhara H, Nakagawa T, Nishimatsu H, Kume H and Homma Y: Multiple primary malignant neoplasms of the glottis, renal pelvis, urinary bladder, oral floor, prostate, and esophagus in a Japanese male patient: A case report. World J Surg Oncol 12: 294, 2014.
36. Mohammed A, Al-Zahrani A, Mansour M, Ghanem H, El Saify A and Hani EK: Triple primary carcinomas: Prostatic adenocarcinoma, bladder urethral carcinoma and papillary thyroid carcinoma: A case report. Am J Cancer Case Rep 3: 24-28, 2015.

37. Testori A, Cioffi U, De Simone M, Bini F, Vaghi A, Lemos AA, Ciulla MM and Alloisio M: Multiple primary synchronous malignant tumors. BMC Res Notes 27: 730, 2015.

38. Pastore AL, Palleschi G, Leto A, Silvestri L, Porta N, Petrozza V and Carbone A: A novel combination of triple metachronous malignancies of the kidney, oropharynx and prostate: A case report. Oncol Lett 10: 917-920, 2015.

39. Adel M, Liao CT, Lee LY, Hsueh C, Lin CY, Fan KH, Wang HM, $\mathrm{Ng} \mathrm{SH}$, Lin CH, Tsao CK, et al: Incidence and outcomes of patients with oral cavity squamous cell carcinoma and fourth primary tumors: A long-term follow-up study in a betel quid chewing endemic area. Medicine (Baltimore) 95: e2950, 2016.

40. Donin N, Filson C, Drakaki A, Tan HJ, Castillo A, Kwan L, Litwin $\mathrm{M}$ and Chamie $\mathrm{K}$ : Risk of second primary malignancies among cancer survivors in the United States, 1992 through 2008. Cancer 122: 3075-3086, 2016.

41. Babacan NA, Aksoy S, Cetin B, Ozdemir NY, Benekli M, Uyeturk U, Ali Kaplan M, Kos T, Karaca H, Oksuzoglu B, et al: Multiple primary malignant neoplasms: Multi-center results from Turkey. J BUON 17: 770-775, 2012.

42. Utada M, Ohno Y, Hori M and Soda M: Incidence of multiple primary cancers and interval between first and second primary cancers. Cancer Sci 105: 890-896, 2014.

43. Yamashita T, Araki K, Tomifuji M, Tanaka Y, Harada E, Suzuki T, Miyamoto S and Shiotani A: Clinical features and treatment outcomes of Japanese head and neck cancer patients with a second primary cancer. Asia Pac J Clin Oncol 13: 172-178, 2017.

44. Hung CY, Ueng SH, Lin YC and Chou WC: Metastatic carcinoma of the urinary bladder in a 67-year-old female with underlying triple primary cancers. J Cancer Res Pract 3: 49-53, 2016.

45. Shiels MS, Gibson T, Sampson J, Albanes D, Andreotti G, Beane Freeman L, Berrington de Gonzalez A, Caporaso N, Curtis RE, Elena J, et al: Cigarette smoking prior to first cancer and risk of second smoking-associated cancers among survivors of bladder, kidney, head and neck, and stage I lung cancers. J Clin Oncol 32: 3989-3995, 2014.

46. TiwanaMS,Hay J,Wu J,WongF,Cheung WandOlsonRA:Incidence of second metachronous head and neck cancers: Population-based outcomes over 25 years. Laryngoscope 124: 2287-2291, 2014.

47. Kundra V: Prostate cancer imaging. Semin Roentgenol 41: $139-149,2006$.

48. Kimura T, Onozawa M, Miyazaki J, Matsuoka T, Joraku A, Kawai K, Nishiyama H, Hinotsu S and Akaza H: Prognostic impact of young age on stage IV prostate cancer treated with primary androgen deprivation therapy. Int J Urol 21: 578-583, 2014.

49. Miller KD, Siegel RL, Lin CC, Mariotto AB, Kramer JL, Rowland JH,Stein KD, Alteri R and Jemal A: Cancer treatment and survivorship statistics, 2016. CA Cancer J Clin 66: 271-289, 2016.

50. Siegel RL, Miller KD and Jemal A: Cancer statistics, 2017. CA Cancer J Clin 67: 7-30, 2017.

51. Strobel K, Haerle SK, Stoeckli SJ, Schrank M, Soyka JD, Veit-Haibach P and Hany TF: Head and neck squamous cell carcinoma (HNSCC)-detection of synchronous primaries with (18)F-FDG PET/CT. Eur J Nucl Med Mol Imaging 36: 919-927, 2009.

52. Kondo N, Tsukuda M and Nishimura G: Diagnostic sensitivity of ${ }^{18}$ fluorodeoxyglucose positron emission tomography for detecting synchronous multiple primary cancers in head and neck cancer patients. Eur Arch Otorhinolaryngol 269: 1503-1507, 2012.

53. Hiraoka A, Hirooka M, Ochi H, Koizumi Y, Shimizu Y, Shiraishi A, Yamago H, Tanihira T, Miyata H, Ninomiya T, et al: Importance of screening for synchronous malignant neoplasms in patients with hepatocellular carcinoma: Impact of FDG PET/CT. Liver Int 33: 1085-1091, 2013.

54. Minamimoto R, Senda M, Terauchi T, Jinnouchi S, Inoue T, Iinuma $\mathrm{T}$, Inoue $\mathrm{T}$, Ito $\mathrm{K}$, Iwata $\mathrm{H}$, Uno K, et al: Analysis of various malignant neoplasms detected by FDG-PET cancer screening program: Based on a Japanese Nationwide Survey. Ann Nucl Med 25: 45-54, 2011.

55. Murakami R, Uozumi H, Hirai T, Nishimura R, Shiraishi S, Ota K, Murakami D, Tomiguchi S, Oya N, Katsuragawa S and Yamashita Y: Impact of FDG-PET/CT imaging on nodal staging for head-and-neck squamous cell carcinoma. Int J Radiat Oncol Biol Phys 68: 377-382, 2007. 